\title{
$(s, S)$ Inventory with Positive Service Time and Retrial of Demands: An Approach through Multiserver Queues
}

\author{
Anoop N. Nair and M. J. Jacob \\ Department of Mathematics, National Institute of Technology Calicut, Calicut, Kerala 673601, India \\ Correspondence should be addressed to Anoop N. Nair; nair.anoopn@gmail.com
}

Received 30 December 2013; Accepted 22 January 2014; Published 17 March 2014

Academic Editors: P. Ekel, R. Varela, and X.-M. Yuan

Copyright ( 2014 A. N. Nair and M. J. Jacob. This is an open access article distributed under the Creative Commons Attribution License, which permits unrestricted use, distribution, and reproduction in any medium, provided the original work is properly cited.

We analyze an $(s, S)$ inventory with positive service time and retrial of demands by considering the inventory as servers of a multiserver queuing system. Demands arrive according to a Poisson process and service time distribution is exponential. On each service completion, the number of demands in the system as well as the number of inventories (servers) is reduced by one. When all servers are busy, new arrivals join an orbit from which they try to access the service at an exponential rate. Using matrix geometric methods the steady state joint distribution of the demands and inventory has been analyzed and a numerical illustration is given.

\section{Introduction}

Inventory models with positive service time were first investigated by Sigman and Simchi-Levi [1]. Thereafter a number of researchers analyzed inventory systems where a queue of demands could be formed. We refer to the survey article of inventory with positive service time by Krishnamoorthy et al. [2] for further details. Many of the results are based on the idea that an inventory is served at a service facility. But in certain cases an arriving demand (customer) itself serves the inventory as in the case of a self-serviceable inventory. To analyze such a model, we consider the inventory as the server which is quite different from the usual multiserver queuing models. When a customer leaves the system after service completion, one server (inventory) also leaves the system which results in the decrement of the total number of servers (inventory) by one. As soon as the inventory reaches a lower level $s$, an order is placed instantaneously and the inventory is restocked to $S$. When the inventory is not available, an arriving customer leaves the system and he tries later for the inventory. To address such a model, the idea of retrial of customers in queuing theory can be used.

A retrial queue is similar to an ordinary queue which has the following additional characteristics. When an arriving customer finds that all servers accessible to him are busy, he joins an infinite buffer called orbit from which he tries to access the server with a given probabilistic or deterministic policy. Thus the formation of a primary queue is not mandatory. The orbit is not an actual physical waiting area, but it simply represents a pool of returning customers. This type of situation arises in many real situations such as telecommunication and computer networks. A retrial queuing system with $c$ servers has been first investigated by Wilkinson [3]. In a multiserver retrial queue with $c>$ 0 servers, when an arriving customer finds a free server, immediately it occupies the server and leaves after service completion. When all the channels are busy, the arriving customers are blocked and they are sent to join the orbit and from the orbit they try to access the servers repeatedly. Such a retrial queue is modeled by a two state Markov process $\{C(t), N(t)\}$ where $C(t)$ represents the number of busy channels and $N(t)$ represents the number of customers in the orbit on the assumption that the interarrival time and service time follow exponential distributions. Thereafter, many different variations of this model have been studied. Neuts and Rao [4] have analyzed a multiserver retrial queue numerically. Kumar et al. [5]. have investigated a multiserver retrial queue with Bernoulli vacation scheduling service. One may refer to Yang and Templeton [6] for review of retrial queue literature. 
Retrial queues with attached inventory have been examined by Krishnamoorthy and Jose [7]. They have considered an inventory system with positive service time and retrial of customers in which an arriving customer encountering the zero inventory level proceeds to an orbit with a probability $\delta$ and is lost forever with probability $1-\delta$. They have succeeded in calculating the expected number of departures after receiving service as well as the expected number of customers lost without getting service. The work of Krishnamoorthy and Islam [8] considers a production inventory with retrial of customers in an $(s, S)$ policy. It is noted that all the models discussed above consider a retrial queue with a single server which services an inventory. A continuous review retrial inventory system with a finite source of customers and identical multiple servers in parallel has been studied by Yadavalli et al. [9].

In this paper, we examine an $(s, S)$ inventory with positive service time and retrial of demands using a multiserver queuing model with retrial of customers where the servers act as an $(s, S)$ inventory without lead time. An arriving customer finds all the servers busy and joins an orbit with infinite capacity from which it tries to access service after a random time. Retrial customers will get service only after at least one replenishment of the inventory. The main difference of this model from a usual multiserver retrial queue is the varying number of servers. Self-service check out inventories have become popular in recent days and are widely used in retail merchandizing as well as online shopping. Any selfserviceable $(s, S)$ inventory with retrial of demands can be analyzed using the model described. Example of such a model can be seen in online shopping where the store procures items according an $(s, S)$ inventory policy and unsatisfied customers attempt to purchase the item as retrials.

The rest of the paper is organized as follows. Section 2 describes the model and Section 3 analyzes the system by finding the stability condition and steady state probabilities. In Section 4 we give some performance measures of the system. We study the inventory cycle time in Section 5 and the cost optimization problem for this model is dealt with in Section 6. Numerical illustrations are given in Section 7.

\section{Model Description}

Consider a multiserver queuing system where an inventory with standard $(s, S)$ policy without lead time acts as servers. Assume that the customers arrive in a Poisson fashion with rate $\lambda$ and the customer gets service whenever there is a free server. The service time of the demand is exponentially distributed with rate $\mu$. After each service completion, the customer as well the server leaves the system so that the total number of available servers is reduced by one. As soon as the level of the inventory reaches $s$, the inventory is replenished to $S$. An arriving customer, who finds that all available servers are busy, joins an infinite buffer called orbit. From the orbit, the customers attempt to access the service at exponentially distributed time intervals with rate $\alpha$. If an incoming orbital customer finds a free server, he joins for service and leaves the system on service completion.

Let $N(t)$ be the number of customers in the orbit at time $t$ and let $A(t)$ be the number of servers in the system, busy as well as idle at time $t$. Let $B(t)$ be the number of busy servers in the system. It is noted that the number of busy servers $B(t)$ is bounded above by $A(t)$. We model this system using a three-dimensional level independent QBD process $\Omega=\{N(t), A(t), B(t)\}$. The queuing system is described by the continuous time stochastic process having state space $E=\{(n, a, b) ; n \geq 0, a=s+1, s+2, \ldots, S$ and $b \leq a\}$. The infinitesimal generator $\Delta$ of the process is given by

$$
\Delta=\left(\begin{array}{cccc}
B & A_{0} & & \\
A_{2} & A_{1} & A_{0} & \\
& A_{2} & A_{1} & A_{0} \\
& & & \cdots \\
& & & \cdots
\end{array}\right)
$$

where the block matrices $B$ and $A_{i}, i=0,1,2$, of order $\sum_{j=1}^{Q}(s+1+j)=(s+1) Q+(Q(Q+1)) / 2$ are described as follows;

$$
\begin{aligned}
& A_{0}=\left(\begin{array}{ccccc}
B_{1} & & & & \\
& B_{2} & & & \\
& & B_{3} & & \\
& & & \ldots & \\
& & & & B_{\mathrm{Q}}
\end{array}\right) \text { with } \\
& B_{j}=\left(\begin{array}{ccccc}
0 & & & \\
& 0 & & & \\
& & 0 & & \\
& & \ldots & \\
& & & & \mu
\end{array}\right)_{(s+1+j) \times(s+1+j)} \\
& B=\left(\begin{array}{ccccc}
C_{1} & & & & E \\
D_{1} & C_{2} & & & \\
& D_{2} & C_{3} & & \\
& & & \ldots & \ldots \\
& & & D_{\mathrm{Q}-1} & C_{\mathrm{Q}}
\end{array}\right) \text { with }
\end{aligned}
$$




$$
\begin{aligned}
& C_{j}=\left(\begin{array}{ccccc}
-\lambda & \lambda & & & \\
& -(\lambda+\mu) & \lambda & & \\
& -(\lambda+2 \mu) & \lambda & \\
& & \cdots & \cdots \\
& & & -(\lambda+(s+j) \mu)
\end{array}\right)_{(s+1+j) \times(s+1+j)} \text { for } j=1,2, \ldots, Q
\end{aligned}
$$

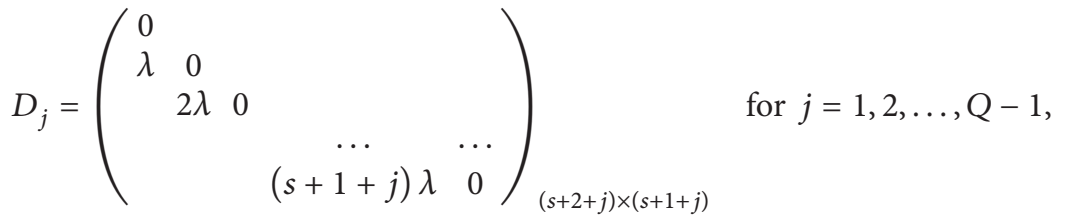

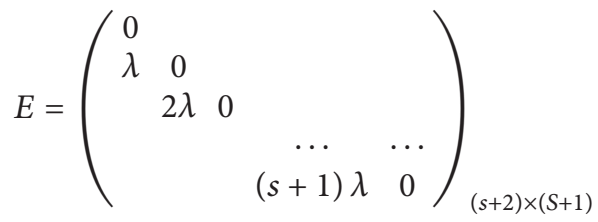

$$
\begin{aligned}
& A_{1}=\left(\begin{array}{ccccc}
C_{1}^{\prime} & & & & E \\
D_{1} & C_{2}^{\prime} & & & \\
& D_{2} & C_{3}^{\prime} & & \\
& & & \cdots & \cdots \\
& & & D_{\mathrm{Q}-1} & C_{\mathrm{Q}}^{\prime}
\end{array}\right) \text { with }
\end{aligned}
$$

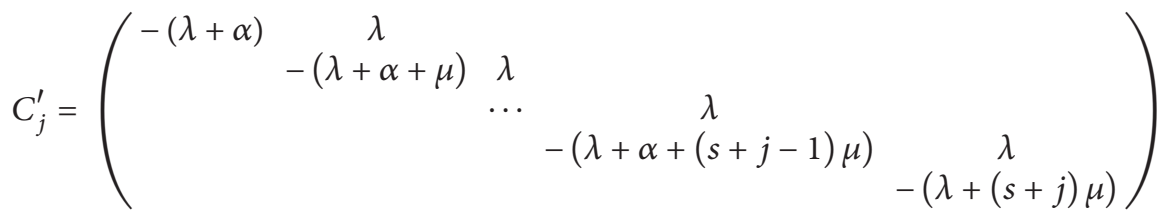

are of order $(s+1+j)$, for $j=1,2, \ldots, Q$ and

$$
\begin{aligned}
& A_{2}=\left(\begin{array}{ccccc}
F_{1} & & & & \\
& F_{2} & & & \\
& & F_{3} & & \\
& & & \ldots & \\
& & & & F_{\mathrm{Q}}
\end{array}\right) \text { with } \\
& F_{j}=\left(\begin{array}{ccccc}
0 & \alpha & & & \\
& 0 & \alpha & & \\
& & \cdots & \ldots & \\
& & & & \alpha \\
& & & & 0
\end{array}\right)_{(s+1+j) \times(s+1+j)} \text { for } j=1,2, \ldots, Q
\end{aligned}
$$

\section{Analysis of the Model}

\subsection{Stability Condition}

Theorem 1. The QBD process described above is stable if and only if

$$
\lambda<\alpha\left(\frac{1-k}{k}\right) \text { with } k=\theta_{(s+1, s+1)}+\theta_{(s+2, s+2)}+\cdots+\theta_{(S, S)} \text {, }
$$

where $\theta_{(i, j)}$ 's are the steady state probabilities of the finite state Markov chain with infinitesimal generator $A=A_{2}+A_{1}+A_{0}$.

Proof. Define $A=A_{2}+A_{1}+A_{0}$ and let $\widetilde{\theta}=\left(\theta_{(s+1,0)}\right.$, $\left.\theta_{(s+1,1)}, \ldots, \theta_{(s+1, s+1)}, \theta_{(s+2,0)}, \ldots, \theta_{(s+2, s+2)}, \ldots, \theta_{(S, 0)}, \ldots, \theta_{(S, S)}\right)$ be the steady state solution of the finite state Markov chain with rate matrix $A$. Then $\widetilde{\theta} A=0$ gives the following system of equations;

$$
\begin{aligned}
& -(\alpha+\lambda) \theta_{(i, 0)}+\mu \theta_{(i+1,1)}=0 \\
& \quad \text { for } i=s+1, s+2, \ldots, S-1 \\
& -(\alpha+\lambda) \theta_{(S, 0)}+\mu \theta_{(s+1,1)}=0 \\
& (\alpha+\lambda) \theta_{(i, j)}-(\alpha+\lambda+(j+1) \mu) \theta_{(i, j+1)} \\
& +(j+2) \mu \theta_{(i+1, j+2)}=0 ; \\
& \quad \text { for } i=s+1, \ldots, S-1, j=0,1, \ldots, i-(s+1) \\
& (\alpha+\lambda) \theta_{(s, j)}-(\alpha+\lambda+(j+1) \mu) \theta_{(s, j+1)} \\
& +(j+2) \mu \theta_{(s+1, j+2)}=0 ; \\
& \quad \text { for } j=0,1, \ldots, s-1
\end{aligned}
$$




$$
\begin{aligned}
& (\alpha+\lambda) \theta_{(i, i-1)}-i \mu \theta_{(i, i)}+(i+1) \mu \theta_{(i+1, i+1)}=0 \text {; } \\
& \text { for } i=s+1, \ldots, S-1 \\
& (\alpha+\lambda) \theta_{(S, j)}-(\alpha+\lambda+(j+1) \mu) \theta_{(S, j+1)}=0 ; \\
& \text { for } j=s, s+1, \ldots, S-2 \\
& (\alpha+\lambda) \theta_{(S, S-1)}-S \mu \theta_{(S, S)}=0,
\end{aligned}
$$

with the normalizing condition

$$
\sum_{i=s+10 \leq j \leq i}^{S} \sum_{(i, j)}=1
$$

$\theta_{(i, j)}$ 's can be obtained by solving the system of linear equations (5) together with (6). The $\mathrm{QBD}$ process with infinitesimal generator $\Delta$ is stable if and only if $\widetilde{\theta} A_{0} e<\widetilde{\theta} A_{2} e$. (see Neuts [10]).

That is, if and only if

$$
\lambda \sum_{i=s+1}^{S} \theta_{(i, i)}<\alpha \sum_{i=s+1}^{S} \sum_{0 \leq j<i} \theta_{(i, j)} .
$$

Applying the normalizing condition (6) we get

$$
\lambda<\alpha\left(\frac{1-k}{k}\right) \text { with } k=\theta_{(s+1, s+1)}+\theta_{(s+2, s+2)}+\cdots+\theta_{(S, S)} .
$$

3.2. Steady State Distributions. Let $\widetilde{\pi}=\left(\widetilde{\pi_{0}}, \widetilde{\pi_{1}}, \widetilde{\pi_{2}}, \ldots\right)$ be the steady state probabilities of the process described where $\widetilde{\pi}_{j}=\left(\pi_{(j, s+1,0)}, \pi_{(j, s+1,1)}, \ldots, \pi_{(j, s+1, s+1)}, \pi_{(j, s+2,0)}, \pi_{(j, s+2,1)}, \ldots\right.$, $\left.\pi_{(j, s+2, s+2)}, \ldots, \pi_{(j, S, 0)}, \ldots, \pi_{(j, S, S)}\right)$. Since $\Delta$ is level independent, its steady state vector is given by

$$
\widetilde{\pi_{j}}=\widetilde{\pi_{0}} R^{j} \quad \text { for } j=1,2, \ldots,
$$

where $R$ is the minimum nonnegative solution of the quadratic expression $R^{2} A_{2}+R A_{1}+A_{0}=0$ (see Neuts [10]) and which can be obtained from the iterative procedure

$$
\begin{aligned}
& R_{(n+1)}=\left(-A_{0}-R_{n}^{2} A_{2}\right) A_{1}^{(-1)} \text { for } n=1,2,3, \ldots \text { with } \\
& R_{1}=0 .
\end{aligned}
$$

To find the boundary vector $\widetilde{\pi_{0}}$, we have from $\tilde{\pi} \Delta=0$, $\widetilde{\pi_{0}} B+\widetilde{\pi_{1}} B=0$ and the normalizing condition $\widetilde{\pi_{0}} e+\widetilde{\pi_{1}} e+$ $\widetilde{\pi_{2}} e+\cdots=1$.

Substituting (9), the above two equations reduce to

$$
\begin{aligned}
& \widetilde{\pi_{0}}\left(B+R A_{2}\right)=0, \\
& \widetilde{\pi_{0}}(1-R)^{-1} e=1 .
\end{aligned}
$$

Thus $\widetilde{\pi_{0}}$ is obtained from (11) and other steady state probabilities can be calculated using (9).

\section{System Performance Measures}

Once the steady state probabilities $\pi_{(i, j, k)}$ for $i=0,1, \ldots$, $j=s+1, s+2, \ldots, S$, and $k=0,1, \ldots, j$ are calculated, we can compute the various performance measures of the system. Some important measures are listed below.

(1) Average number of customers in the orbit $\left(E_{o}\right)$ :

$$
E_{o}=\sum_{i=0}^{\infty} \sum_{j=s+1}^{S} \sum_{k=0}^{j} i \pi_{(i, j, k)} .
$$

(2) Average number of available (busy as well as idle) servers $\left(E_{a}\right)$ :

$$
E_{a}=\sum_{i=0}^{\infty} \sum_{j=s+1}^{S} \sum_{k=0}^{j} j \pi_{(i, j, k)} .
$$

(3) Average number of busy servers $\left(E_{b}\right)$ :

$$
E_{b}=\sum_{i=0}^{\infty} \sum_{j=s+1}^{S} \sum_{k=0}^{j} k \pi_{(i, j, k)} .
$$

(4) Probability that the system is busy (probability that arriving customers are sent to orbit $)\left(P_{\text {busy }}\right)$ :

$$
P_{\text {busy }}=\sum_{i=0}^{\infty} \sum_{j=s+1}^{S} \pi_{(i, j, j)} \text {. }
$$

(5) Probability that an arriving customer enters the service without waiting $P_{\text {ser }}$ :

$$
P_{\text {ser }}=1-\sum_{i=0}^{\infty} \sum_{j=s+1}^{S} \pi_{(i, j, j)}=1-P_{\text {busy }} .
$$

(6) First hitting time.

We define the first hitting time as the time until an arriving customer goes to the orbit for the first time given that the system starts from the state $(0, S, 0)$ at time 0 . This may be regarded as the sojourn time in the retrial state 0 and we denote this by $\tau_{f}$. We analyze the first hitting time as the time until the absorption of a finite state Markov chain $Z(t)$ with state space $\{(i, j) ; j=0,1,2, \ldots, i$ and $i=s+$ $1, s+2, \ldots, S\} \bigcup\left\{\xi_{f}\right\}$, where $\xi_{f}$ is the absorbing state. The rate matrix of the process is given by the matrix

$$
\Delta_{f}=\left(\begin{array}{cc}
T_{f} & T_{f}^{\prime} \\
\tilde{0} & 0
\end{array}\right),
$$

where $T_{f}=B$ as in $\Delta$ is described in Section 2 with dimension $\sum_{j=1}^{\mathrm{Q}}(s+1+j)$ and $T_{f}^{\prime}=(0,0, \ldots, \lambda, 0, \ldots, \lambda)^{\prime}$, where $\lambda$ 's are at positions $s+1+j$, for $j=1,2, \ldots, S$.

Let $\widetilde{\pi_{f}}=(0, \ldots, 1,0, \ldots)$ be the initial distribution of $Z(t)$, where 1 corresponds to the state $(S, 0)$; then $\tau_{f}$ follows a phase type distribution with distribution function $F(t)=$ $1-\widetilde{\pi_{f}} e^{-T_{f} t} e$. 
Remark 2. When the system is in state $(n, i, i) ; n \geq 0$ and $s+1 \leq i \leq S$, all servers will be busy. Once this state is achieved, external arrivals directly enter the orbit for a period of $i-s$ service completions. Therefore the time taken from the instant that the system reaches the state $(n, i, i)$ to the next inventory replenishment time follows a generalized Erlang distribution of order $i-s$ with parameters $\{i \mu,(i-1) \mu, \ldots,(s+$ 1) $\mu\}$.

\section{Inventory Recycle Time}

An inventory recycle time can be defined as the time between two consecutive replenishments, that is, the time taken to reach $S$ for the first time starting from a state $(k, S, i) ; k \geq 0$, $i \leq S$, is defined as a recycle time. Clearly the recycle time is a random variable which depends on both the number of customers in the orbit $k$ and number of busy servers $i$.

We can obtain the recycle time by considering the time until the absorption of a finite state Markov chain (MC). It may be noted that the future arrivals to the orbit do not affect the recycle time once all the available servers are busy starting from a state $(k, S, i)$. Thus if $k \geq S$, the recycle time will depend only on $i$ and we denote it by $\tau_{i}$. But if $k<S$, the recycle time will depend on both $i$ and $k$; therefore we denote it by $\tau_{k, i}$. Let $\delta$ be the absorbing state of the Markov chain, which absorbs the states $(k, s+1, i)$ with $i>0$ at a service completion. The state space of the finite state Markov chain is given by

$$
E^{\tau}=\left\{\begin{array}{r}
\{(n, a, b)\} \bigcup\{\delta\} ; 0 \leq n \leq k, s+1 \leq a \leq S, \\
0 \leq b \leq a ; \quad \text { if } k \geq S . \\
\{(n, a, b)\} \bigcup\{\delta\} ; k-Q \leq n \leq k, s+1 \leq a \leq S, \\
0 \leq b \leq a ; \quad \text { if } k<S .
\end{array}\right.
$$

We arrange the state space of the above MC in the usual lexicographic order. Let $\widetilde{P}=(0,0, \ldots, 1, \ldots, 0)$ be a state matrix, where 1 corresponds to the state $(k, S, i)$, from which the process starts. The possible transitions of the $\mathrm{MC}$ are given below (Table 1).

Now we consider the following cases.

(1) If $k \geq S$. The rate matrix of the finite state MC takes the form

$$
Q_{i}=\left(\begin{array}{cc}
T_{i} & T_{i}^{\prime} \\
\tilde{0} & 0
\end{array}\right)
$$

where $T_{i}^{\prime}$ is a column matrix corresponding to the absorbing state and $T_{i}$ is the rate transition matrix. Thus the recycle time follows a phase type distribution with the distribution function

$$
P\left(\tau_{i}<t\right)=1-\widetilde{P} e^{-T_{i} t} e .
$$

The average inventory recycle time starting from the state $(k, S, i)$ is given by

$$
E\left(\tau_{i}\right)=-\widetilde{P}\left(T_{i}\right)^{-1} e
$$

TABLE 1: State transitions of the finite state MC.

\begin{tabular}{lccc}
\hline From & To & Rate & \\
\hline$(n, a, b)$ & $(n, a, b+1)$ & $\lambda$ & $b<a$ \\
$(n, a, b)$ & $(n-1, a, b+1)$ & $\alpha$ & $1 \leq n, b<a$ \\
$(n, a, b)$ & $(n, a-1, b-1)$ & $b \mu$ & $1 \leq b<a$ \\
$(n, b, b)$ & $(n, b-1, b-1)$ & $b \mu$ & $s+1<b \leq S$ \\
$(n, s+1, b)$ & $\delta$ & $b \mu$ & $1 \leq b$ \\
\hline
\end{tabular}

(2) If $k<S$. The rate matrix of the finite state MC takes the form

$$
Q_{k, i}=\left(\begin{array}{cc}
T_{k, i} & T_{k, i}^{\prime} \\
\widetilde{0} & 0
\end{array}\right)
$$

where $T_{k, i}^{\prime}$ is a column matrix corresponding to the absorbing state and $T_{k, i}$ is the rate transition matrix. Thus the recycle time follows a phase type distribution with the distribution function

$$
P\left(\tau_{k, i}<t\right)=1-\widetilde{P} e^{-T_{k, i} t} e .
$$

The average inventory recycle time starting from the state $(k, S, i)$ is given by

$$
E\left(\tau_{k, i}\right)=-\widetilde{P}\left(T_{k, i}\right)^{-1} e .
$$

Thus the expected recycle time of the system is given by the following equation:

$$
E(\tau)=\sum_{k=0}^{S-1} \sum_{i=0}^{S} E\left(\tau_{k, i}\right) \pi_{(k, S, i)}+\sum_{k=S}^{\infty} \sum_{i=0}^{S} E\left(\tau_{i}\right) \pi_{(k, S, i)} .
$$

\section{An Optimization Problem for $M / M /(s, S)$ Retrial Model}

In the model discussed, servers are nothing but inventories which follow standard $(s, S)$ policy. Therefore we can consider a probabilistic cost optimization problem. Based on the performance measures we construct a cost function as follows:

$$
T C=E_{o} * C_{w}+E_{a} * C_{h}+\frac{C_{o}}{E(\tau)},
$$

where $C_{w}$ is the waiting cost of a customer in the orbit per unit time, $C_{h}$ is the holding cost of a server (inventory) in the system per unit time, and $C_{o}$ is the ordering cost per order. The cost function above is used to find the optimum reorder level $s$ that will minimize the total cost.

\section{Numerical Illustrations}

Now we provide some numerical illustrations. Table 2 gives the steady state probabilities of the model $M / M /(1,3)$ with $\lambda=2, \mu=3$ and $\alpha=2$. Various performance measures for different values of the reorder level $s$ with $\lambda=2, \mu=3$, and $\alpha=2$ are shown in Table 3. Figure 1 gives the optimum order quantity $s$ of the system with $\lambda=2, \mu=3, \alpha=2, C_{w}=10$, $C_{h}=2$, and $C_{o}=0$. It can be observed that the minimum cost is obtained when the reorder level $s=2$. 
TABLE 2: Stationary distributions of $M / M /(1,3)$ retrial system.

\begin{tabular}{ccccc}
\hline$n$ & $(2,0)$ & $(2,1)$ & $(2,2)$ & $(3,0)$ \\
\hline 0 & 0.3548130 & 0.1175023 & 0.0196339 & 0.3525071 \\
1 & 0.0037211 & 0.0013867 & 0.0034112 & 0.0013867 \\
2 & 0.0006355 & 0.0002363 & 0.0005828 & 0.0002363 \\
3 & 0.0001076 & 0.0000399 & 0.0000987 & 0.0000399 \\
4 & 0.0000181 & 0.0000067 & 0.0000166 & 0.0000067 \\
5 & 0.0000030 & 0.0000011 & 0.0000028 & 0.0000011 \\
6 & 0.0000005 & 0.0000002 & 0.0000005 & 0.0000002 \\
7 & 0.0000001 & 0.0000000 & 0.0000001 & 0.0000000 \\
\hline$n$ & $(3,1)$ & $(3,2)$ & $(3,3)$ & \\
\hline 0 & 0.1182710 & 0.0179590 & 0.0019068 & \\
1 & 0.0037211 & 0.0005547 & 0.0002651 & \\
2 & 0.0006355 & 0.0000945 & 0.0000392 & \\
3 & 0.0001076 & 0.0000160 & 0.0000061 & \\
4 & 0.0000181 & 0.0000027 & 0.0000010 & \\
5 & 0.0000030 & 0.0000005 & 0.0000002 & \\
6 & 0.0000005 & 0.0000001 & 0.0000000 & \\
7 & 0.0000001 & 0.0000000 & 0.0000000 & \\
& & & &
\end{tabular}

TABle 3: Performance measures of $M / M /(s, 4)$ retrial queue.

\begin{tabular}{cccccc}
\hline$s$ & $E_{o}$ & $E_{a}$ & $E_{b}$ & $P_{\text {busy }}$ & $E\left(\tau_{f}\right)$ \\
\hline 0 & 0.8542 & 2.6634 & 1.1987 & 0.2993 & 4.6403 \\
1 & 0.1371 & 3.4787 & 1.4966 & 0.1924 & 11.8358 \\
2 & 0.0284 & 3.5005 & 0.6667 & 0.0177 & 38.9741 \\
3 & 0.0064 & 4 & 0.6667 & 0.0044 & 143.8750
\end{tabular}

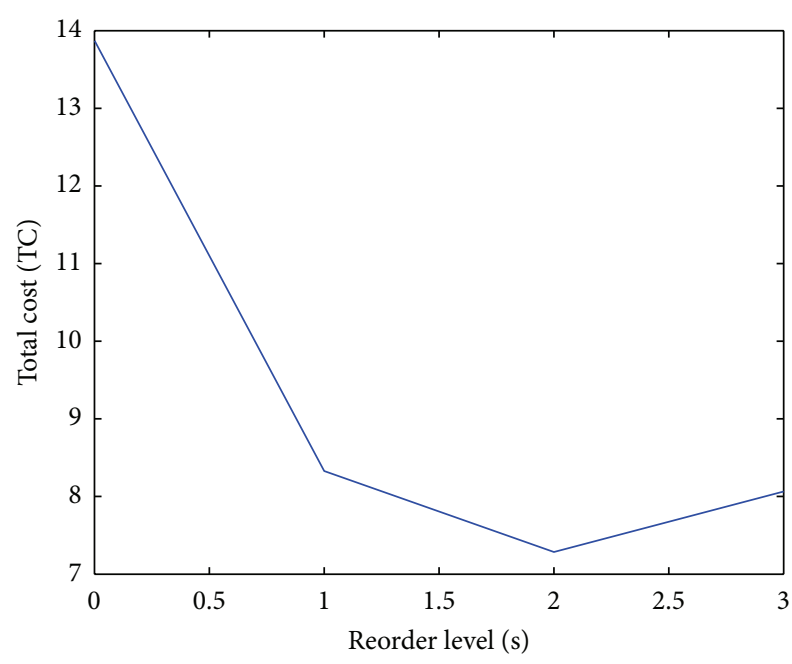

Figure 1: Effect of reorder level on Total cost.

\section{Conclusion}

In this paper, we have analyzed a multiserver retrial queue where the servers act as an $(s, S)$ inventory. Primary interarrival times as well as the orbital inter arrival times follow exponential distributions with constant rates. We have derived the steady state distribution of the system using matrix analytic methods and several performance measures have also been calculated. The cost analysis of this system is very significant because the servers in the system are considered as inventory so that different costs such as holding cost and shortage cost are involved. We have performed the cost analysis with respect to the reorder level. Numerical solutions are presented to illustrate the qualitative behavior of the system.

\section{Conflict of Interests}

The authors declare that there is no conflict of interests regarding the publication of this paper.

\section{Acknowledgments}

We thank the anonymous referees for their detailed comments and helpful suggestions.

\section{References}

[1] K. Sigman and D. Simchi-Levi, "Light traffic heuristic for an M/G/1 queue with limited inventory," Annals of Operations Research, vol. 40, no. 1, pp. 371-380, 1992.

[2] A. Krishnamoorthy, B. Lakshmy, and R. Manikandan, "A survey on inventory models with positive service time," OPSEARCH, vol. 48, no. 2, pp. 153-169, 2011.

[3] R. I. Wilkinson, "Theories for toll trac engineering in the U.S.A," Bell Systems Technical Journal, vol. 35, no. 2, pp. 421-507, 1956.

[4] M. F. Neuts and B. M. Rao, "Numerical investigation of a multiserver retrial model," Queueing Systems, vol. 7, no. 2, pp. 169-189, 1990.

[5] B. K. Kumar, R. Rukmani, and V. Thangaraj, "An M/M/C retrial queueing system with Bernoulli vacations," Journal of Systems Science and Systems Engineering, vol. 18, no. 2, pp. 222-242, 2009.

[6] T. Yang and J. G. C. Templeton, "A survey on retrial queues," Queueing Systems, vol. 2, no. 3, pp. 201-233, 1987.

[7] A. Krishnamoorthy and K. P. Jose, "An $(s, S)$ inventory system with positive lead time, loss and retrial of customers," Stochastic Modelling and Applications, vol. 8, no. 2, p. 5671, 2005.

[8] A. Krishnamoorthy and M. E. Islam, "Production inventory with retrial of customers in an $(s, S)$ policy," Stochastic Modelling and Applications, vol. 6, no. 2, p. 111, 2003.

[9] V.S. S. Yadavalli, B. Sivakumar, G. Arivarignan, and O. Adetunji, "A finite source multi-server inventory system with service facility," Computers \& Industrial Engineering, vol. 63, no. 4, pp. 739-753, 2012.

[10] M. F. Neuts, Matrix-Geometric Solutions in Stochastic ModelsAn Al-Gorithmic Approach, The Johns Hopkins University Press, 1981. 


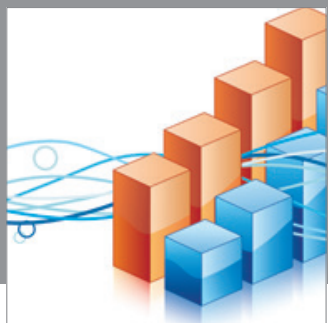

Advances in

Operations Research

mansans

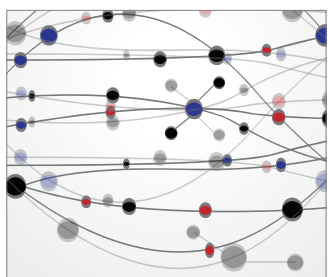

The Scientific World Journal
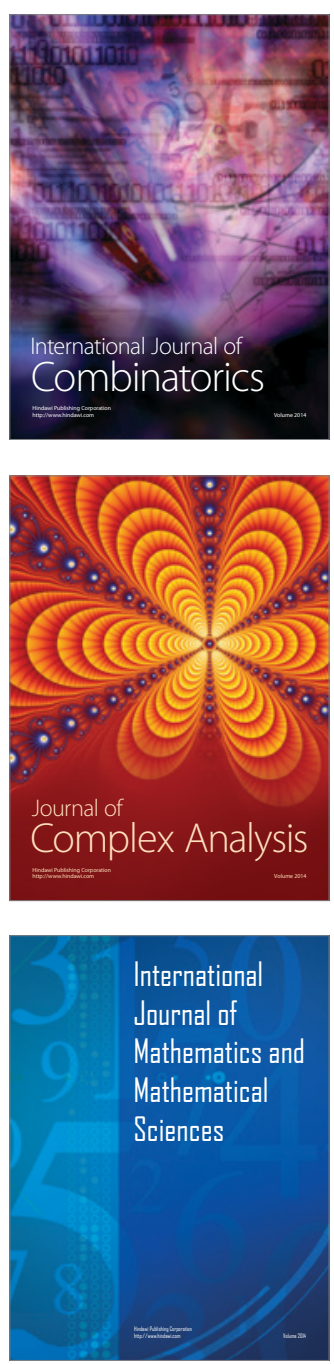
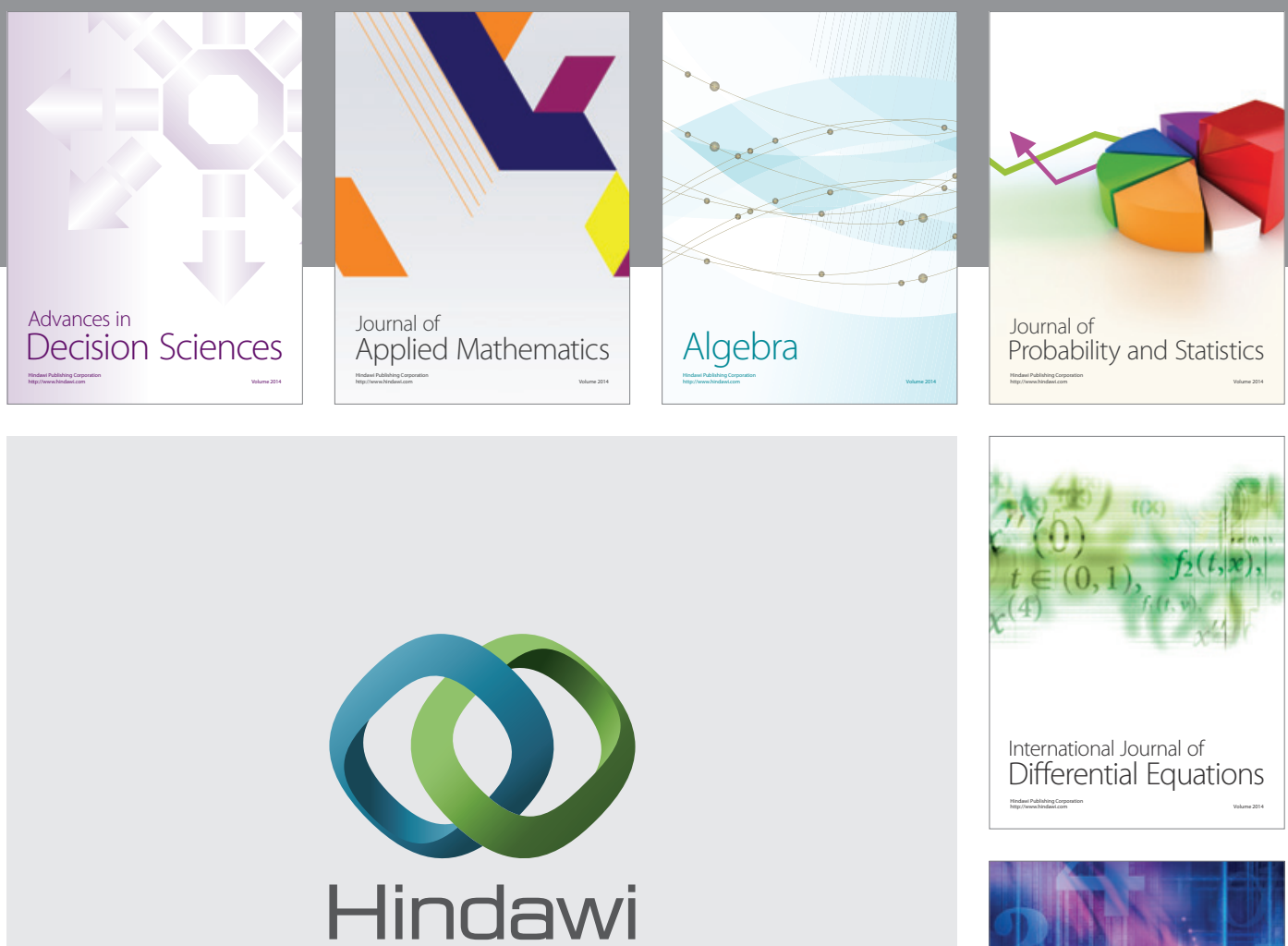

Submit your manuscripts at http://www.hindawi.com
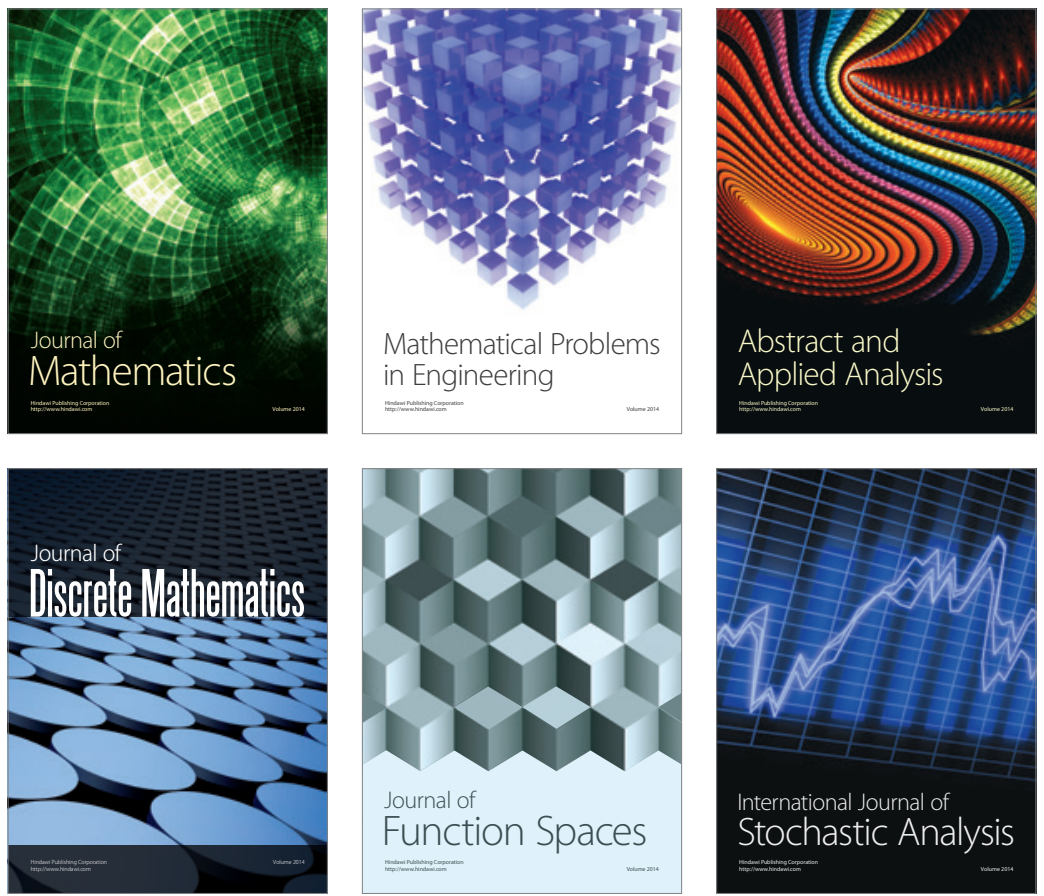

Journal of

Function Spaces

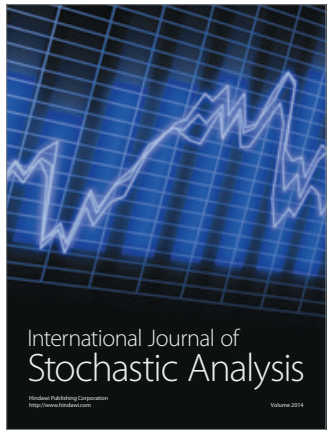

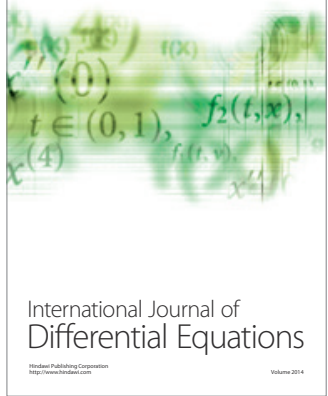
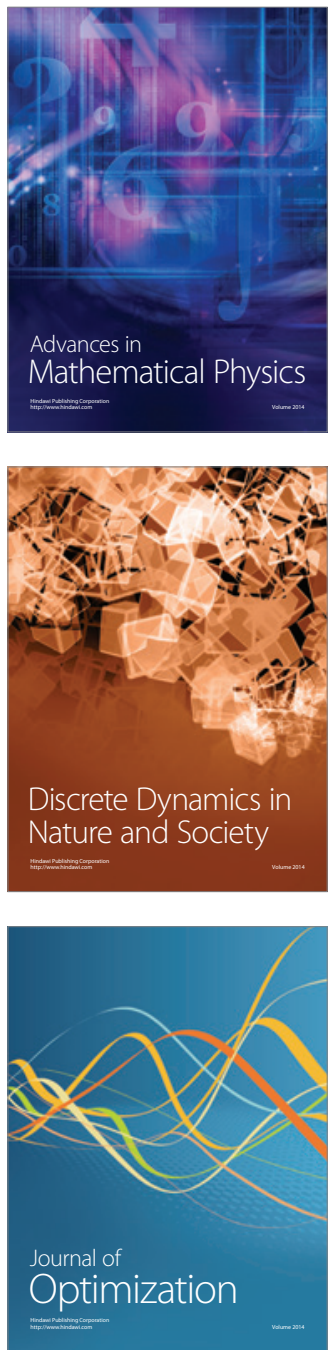